\title{
Sylvia Wynter y el problema del humanismo ${ }^{1}$
}

\author{
Sylvia Wynter and the problem of “humanism” \\ Luis Adrián Mora Rodríguez \\ Universidad de Costa Rica, San José, Costa Rica \\ luis.morarodriguez@ucr.ac.cr
}

Resumen: Sylvia Wynter es una de las pensadoras más fecundas del Caribe. Si bien su obra se ha desarrollado en inglés, el interés de sus trabajos ha roto siempre las barreras impuestas por la presencia imperial. Especialista de la literatura del Siglo de Oro español, Wynter no ha cesado de reflexionar sobre los aspectos históricos e intelectuales que marcan el surgimiento del Caribe en la conciencia moderna, así como sobre la emergencia de una cierta definición de lo humano. Wynter pertenece a lo que podríamos llamar la primera generación de la autodeterminación y la independencia insular caribeña. Su recorrido intelectual está íntimamente aparejado con su trayectoria personal y social. En este artículo, abordaré la crítica sistemática que Wynter hace a las distintas versiones históricas e intelectuales

1 Este texto nace como resultado de una investigación sobre las nociones de historia, identidad y poder en tres autores caribeños: Sylvia Wynter, Édouard Glissant y Antonio Benítez-Rojo. Esta investigación forma parte del Proyecto "Connected Worlds: The Caribbean, Origin of Modern World". Dicho proyecto es financiado por la Unión Europea, en el marco del Programa Horizonte 2020 Investigación e Innovación, Marie Sklodowska Curie, Grant Agreement No 823846. Proyecto dirigido por la Profesora Consuelo Naranjo Orovio, Instituto de Historia del CSIC. 
del "humanismo 2". Mi objetivo es mostrar cómo dicha crítica postula un funcionamiento sistemático y binario del ordenamiento social contemporáneo, heredado de la historia imperial occidental. Cómo se sostiene dicha crítica y cuáles son sus posibles límites es lo que me interesa desarrollar en las siguientes líneas.

Palabras clave: humanismo, caribe, historia, conquista, colonización.

Aвstract: Sylvia Wynter is one of the most important thinkers in the Caribbean. Although her work has been developed in English, she has always broken down the barriers imposed by the imperial presence. A specialist in Spanish Golden Age literature, Wynter has not ceased to reflect on the historical and intellectual aspects that mark the emergence of the Caribbean in modern consciousness, as well as on the emergence of a certain definition of the human. Wynter belongs to what we might call the first generation of an independent and self-determined Caribbean. Her intellectual journey is intimately linked to her personal and social trajectory. In this article we will address Wynter's systematic criticism of the different historical and intellectual versions of "humanism". My objective is to show how this critique postulates a systematic and binary functioning of the contemporary social order, inherited from Western imperial history. How this criticism is sustained and what are its possible limits is what I am interested in developing in the following lines.

KEYwORDs: humanism, caribbean, history, conquest, colonization.

2 El término "humanismo" refiere aquí a dos sentidos claramente definidos por Wynter. Un primer sentido, que la autora señala como "humanismo cívico", es aquella visión del hombre establecida por los intelectuales renacentistas. Visión que tiene como marca una autonomía de lo humano con respecto a la esfera religiosa. El ser humano se define como un ser cuya existencia está ordenada en relación con la esfera política. Se trata de un ser que puede ahora intervenir sobre la naturaleza, descubrir sus leyes, puesto que el mundo es una creación para él. Representantes de este humanismo son intelectuales como Pico Della Mirandola o Copérnico. Este humanismo se transforma en el siglo XIX con la teoría de la evolución de Charles Darwin. A partir de aquí, Wynter reconoce un segundo sentido del término humanismo, refiriéndose a la forma de definir lo humano como producto de la evolución, estableciendo así una definición basada en la biología y completamente secular. El esfuerzo teórico de Wynter consiste en mostrar los límites de estas dos versiones del humanismo. En el artículo, cuando me refiero de manera general al término humanismo estaré haciendo alusión a ambas versiones. Al entrar en el análisis detallado, distinguiré entre "humanismo cívico" y "humanismo evolucionista". 


\section{INTRODUCCIÓN}

El Caribe ha sido históricamente un lugar privilegiado para la interrogación sobre lo humano. Cuando los revolucionarios haitianos redactaron su constitución, en 1804, retomaron y radicalizaron la definición de lo humano, a la vez que cuestionaron las bases políticas sobre las cuales se construía el mundo moderno. En el siglo XX, el Caribe fue el terreno de la interrogación estético-política de la négritude que, desarrollándose sobre las ruinas de un continente destrozado por la guerra, retó a Europa a enfrentar su realidad imperial. Los movimientos de la descolonización encontraron en el Caribe también un espacio fértil para interrogar la definición de lo humano, así como los grandes valores afirmados por el humanismo, como la libertad y la igualdad o la racionalidad.

El Caribe sirve entonces de espejo a una serie de interrogantes: ¿qué es lo que nos hace humanos?, ¿es acaso el control de nuestras pasiones o, más bien nuestra inteligencia superior?, ¿se trata de la certidumbre de estar en la cúspide de la evolución? Estas preguntas organizan la crítica que Sylvia Wynter desarrolla en su obra. En efecto, se trata de preguntas que tanto la filosofía como la ciencia han pretendido responder, y es sobre las respuestas a ellas que se construye el mundo en el que vivimos. El panorama actual de devastación planetaria y de desigualdad exacerbada exige, por lo tanto, interrogar cómo se han planteado dichas preguntas y qué se esconde a nuestros ojos en su formulación. Este trabajo de identificación y ordenamiento es el que Sylvia Wynter propone. Con el fin de acercarnos a su crítica, propongo a continuación un análisis en dos tiempos. En primer lugar, me enfocaré rápidamente en el recorrido intelectual y personal de Wynter, con el fin de señalar algunos puntos que considero importantes para el desarrollo de su crítica al humanismo. En segundo lugar, analizaré los componentes de esta crítica a través del estudio de varios de sus ensayos de los años noventa y principios del siglo XXI.

\section{UNA TRAYECTORIA CARIBEÑA}

Wynter nace en Cuba, de padres jamaiquinos, en 1928. Sus padres se habían ido a la isla para trabajar en la zafra azucarera. De muy escasos 
recursos, logra sin embargo completar la educación básica de vuelta a Jamaica. Y gracias al apoyo de un gran maestro, Bruce Wardropper, comienza sus estudios superiores en Londres, a partir de 1947. Para ese entonces, la ciudad británica es el centro de un todavía poderoso imperio. Es allí, según lo cuenta Wynter en una entrevista con David Scott (126), que ella se consume en un universo paradójicamente "caribeńo". Es decir, en la capital imperial es donde surge la conciencia de pertenecer a las "Indias Occidentales". En este Londres de posguerra se reúnen jóvenes estudiantes de diferentes islas caribeñas que han sido históricamente separadas por la geopolítica imperial. Es allí donde comienza a tejerse la certidumbre de la unidad caribeña en contradicción con la narrativa británica. Nos dice Wynter: "In the different islands we had been totally cut off from each other. We weren't even taught Caribbean geography in the schools. The geography that was taught was that of England. The history that was taught was English history"3 (Scott 129).

Es en Londres, donde aquellos que fueron formados como "ciudadanos británicos", "sujetos imperiales", se dan cuenta de que en realidad son "nativos". Es decir, que representan -por el color de su piel y su procedencia- lo inverso del ideal ciudadano imperial. Es a partir de esta nueva caracterización como "otredad" que los caribeños en la metrópoli buscarán autodefinirse y expresarse. Wynter señala que es la primera vez que se atreven a "hablar"; es decir, que se atreven a crear a partir de sus propias experiencias y especificidades. Así aparece la particularidad de su situación y es cuando los paradigmas del humanismo en el cual habían sido educados comienzan a agrietarse (Scott 129).

De esta manera, el retorno de Wynter a Jamaica, a finales de los ańos cincuenta, significa el inicio de su compromiso anticolonial y su reflexión sobre las formas de entender y comprender que bosquejan nuestra relación con la realidad. Su pensamiento comienza a construirse desde el ámbito estético y político. Una de las inquietudes que lo anima es la construcción de lo "nacional"; es decir, la posibilidad, ahora como isla independiente, que tiene Jamaica de construirse como Estado-nación ${ }^{4}$.

3 "En las diferentes islas habíamos quedado completamente aislados unos de otros. $\mathrm{Ni}$ siquiera nos enseñaban geografía caribeña en las escuelas. La geografía que se enseñaba era la de Inglaterra. La historia que se enseñaba era la de Inglaterra” (Traducción del editor).

4 Esta interrogación sobre "a nación" es una pregunta frecuente en la reflexión caribeña. Autores como Antonio Benítez-Rojo (54) la han sistematizado señalando 
Es desde esta perspectiva que Wynter va a interesarse por las discusiones en torno a una literatura nacional, a un arte nacional. Para Jamaica, excolonia británica, la necesidad de construir su propia cultura es una cuestión fundamental, puesto que todo el legado colonial se transmite a partir de la educación oficial. Así, por ejemplo, la narrativa histórica del Imperio Británico considera traidores y asesinos a aquellos nativos jamaiquinos que lucharon contra el colonizador inglés (Scott 138; Braithwaite 59). Dicho aprendizaje marca a generaciones enteras, de ahí la necesidad de reapropiarse de la historia y, claro está, del arte y la cultura en general.

La influencia de otra intelectual caribeña, Elsa Goveia, es aquí importante. Wynter la conoce en Londres y lee con detenimiento los Estudios de historiografía de las Antillas Inglesas hasta finales del siglo XIX. Esta obra de Goveia es considerada como un pilar de la historiografía caribeña de los años sesenta. La complejidad de las sociedades caribeńas, inmersas en la dinámica de la modernidad desde sus inicios, marcadas por la esclavitud a gran escala y por las luchas imperiales, hace necesario un abordaje nuevo. Se trata de romper con perspectivas aprendidas, que repiten los patrones de dominación (Goveia 135). Se trata también de reconocer, más allá del modelo del Estado-nación, realidades sociopolíticas originales que desafían la fragmentación insular y la dependencia metropolitana. Goveia aporta igualmente una reflexión sobre el orden social racial. Es en el Caribe donde se instala primero lo que más adelante autores como Du Bois van a analizar en términos de diferenciación racial sistematizada (161). Una separación radical que enmarca el terreno de lo económico, lo social y, por supuesto, lo político. Nos dice Wynter:

I think Elsa Goveia was the one who put it accurately when she said that it was only in the context of the anticolonial movement that all of a sudden writers began writing, painters began painting, that people who has been silent for so long now "found new voices"s (Scott 128).

que se trata de una ruta sin salida. Como se verá más adelante, es la presencia de un esquema repetitivo de organización social y económica, "la Plantación", que permite, en parte, superar esta ruta de la "ación"

5 "Creo que fue Elsa Goveia quien lo puso más precisamente cuando dijo que fue solo en el contexto del movimiento anticolonial que, de la noche a la mañana, los escritores comenzaron a escribir, los pintores a pintar y la gente, 
Wynter retoma estas premisas en sus textos de finales de los años sesenta y principios de los setenta, en los cuales formula concepciones importantes de su pensamiento, como la "poética del desencanto", la crítica del humanismo en el contexto de la plantación esclavista y de la expansión colonial, así como el análisis del poder que tienen las estructuras de conocimiento para ordenar procesos históricos y sociales. En esta fase, Wynter critica una serie de conceptos que pretenden explicar las sociedades caribeñas posindependencia (Edwards 15). Así, por ejemplo, publica un artículo sobre El diario de Lady Nugent donde ataca el mito de la armonía racial. Wynter considera este mito como un síntoma del rechazo social frente al pasado esclavista ("Lady Nugent's" 28). Para ella, enfrentar el pasado colonialista y racista que marca aún a las nuevas sociedades caribeñas formalmente independientes es un requisito para poder efectuar una verdadera crítica estética y social. En efecto, el peso de la experiencia colonial se vive también en el ámbito de la producción cultural, literaria y artística (Wynter, "Jonkonnu in Jamaica" 40). La crítica cultural en las Antillas debe alejarse de la lectura idealista, heredada del canon europeo. De esta manera, el trabajo de crítica literaria de Wynter se separa de los modelos anglosajones para acercarse más a la teoría crítica de Adorno. Es hacia la sociocrítica que se mueve su trabajo, considerando que el discurso sobre la literatura no puede ser solamente un discurso estético, sino que tiene que ver también con la historia y la cultura (Edwards 18). Se trata de una forma de leer las relaciones sociales conflictivas del Caribe. En efecto, ocultar la posición colonial de los críticos es hacer abstracción de las relaciones sociales que los constituyen como tales. Es a la vez imperativo, desde la producción intelectual, señalar las narrativas políticas que, bajo el manto de la "unidad racial" fomentan falsos populismos (Wynter, "One love” 70).

Este trabajo de análisis histórico y de reflexión estética puede verse expresado, por ejemplo, en el ensayo "Novel and History, Plot and Plantation”, en el que Wynter piensa la construcción del orden social a partir del esquema moderno de la plantación. Para la autora, la plantación representa la manifestación física, geográfica de la modernidad, como máquina capitalista instalada en el espacio virgen de la colonia.

que hasta entonces había guardado silencio por mucho tiempo, 'encontró una nueva voz"' (Traducción del editor). 
Una máquina unida al sistema de explotación mundial, engarzada con una lógica de segregación e inferiorización raciall ("Novel” 98). Así, por ejemplo, Wynter explica:

The plantation system which, under the liberal free trade rhetoric which freed the slaves, compensated the masters and set the slaves in a world dominated by market relations, to fend naked for themselves, was the first sketch of monopoly capitalism? ("Novel” 95).

Se trata además de una reflexión que, en la línea de autores como Eric Williams, retoma la importancia del Caribe como "área de plantación". De esta manera, entender la constitución de las sociedades caribeñas es observar cómo históricamente estas sociedades fueron creadas e instituidas de la mano del capitalismo mercantil y la economía de mercado. Afirma Wynter:

our societies were both cause and effect of the emergence of the market economy; an emergence which marked a change of such world historical magnitude, that we are all, without exception, still "enchanted", imprisoned, deformed and schizophrenic in its bewitched reality ${ }^{8}$ (ibid.).

Esta idea que conecta lo sucedido en el Caribe con la transformación de la modernidad es una idea presente en gran número de autores caribeños, tanto del Caribe hispanohablante-como Fernández Retamar y Benítez Rojo-, como en el Caribe francófono -en el planteamiento de

6 El acercamiento con la concepción de Antonio Benítez-Rojo es aquí imperativo. Benítez-Rojo considera entonces la forma de la Plantación (con mayúscula) como la dinámica preponderante, no solo en el ordenamiento económico, sino también en la génesis social del Caribe. Lo que del Caribe "se repite" es precisamente la máquina de la Plantación (Benítez Rojo 50).

7 "El sistema de las plantaciones, que, bajo la retórica liberal de libre comercio con que liberaba a los esclavos, compensaba a los amos y dejaba a los esclavos -en un mundo dominado por las relaciones de mercado- desnudos y a su suerte, fue el primer boceto del capitalismo de monopolio" (Traducción del editor).

8 "Nuestras sociedades fueron tanto causa como efecto de la emergencia de la econom'a de mercado. Una emergencia que significó un cambio de tal magnitud histórica que todos seguimos todavía, sin excepción, 'encantados', aprisionados, deformados y esquizofrénicos por su realidad embrujada” (Traducción del editor). 
Césaire, Fanon, Glissant-y anglófono -por parte de intelectuales como Williams y Walcott-. En ellos podemos encontrar una reflexión sobre la centralidad que tiene la región, en términos económicos y políticos, para el surgimiento de la modernidad y la consolidación de Occidente como potencia. Estos trabajos interrogan además el entramado social que se impone en el Caribe desde la llegada de Colón, junto a la destrucción de los pueblos autóctonos y la importación masiva y brutal de las poblaciones negras esclavizadas. Son estos elementos los que le permiten a Wynter desarrollar una visión generalizada de la cuestión de lo humano. En efecto, ¿cómo se construye la posibilidad de esclavizar al Otro?, ¿qué significa ese espacio de violencia que la plantación representa?, ¿`ómo sobreviven estos estereotipos y prácticas racistas hasta hoy? Y, sobre todo, ¿qué posibilidad tenemos de vencer dichas prácticas?

Estas interrogantes parecen ordenar el trabajo de Wynter a partir de los años setenta. Un elemento central en el desarrollo de su reflexión tiene que ver con su trabajo universitario en Estados Unidos. Luego de su llegada a la Universidad de California, en San Diego, el año 1974, Wynter desarrolla los pilares de lo que será su crítica a las distintas figuras de lo humano. La experiencia de la segregación racial y los movimientos sociales de los negros estadounidenses tendrán un fuerte impacto en la lectura crítica que Wynter hace de esta idea del humanismo, como lo expondré a continuación.

\section{El PROBLEMA DEL HUMANISMO}

Para entender cómo Wynter define y estudia el "humanismo", es necesario comprender cómo este surge y se instala. Wynter destaca que cada sociedad posee y reproduce un principio de entendimiento y justificación de sí misma y del universo. Este principio de ordenamiento está indexado, definido por una entidad sobrenatural y dicha entidad es la que otorga las categorías para el orden social. Dichas categorías definen también los criterios de lo humano (Wynter, "1492: Una nueva visión” 367). Es decir, toda sociedad histórica hace mediar las categorías que ordenan su funcionamiento a través de una entidad imaginada como supracultural y sobrenatural; es decir, una o varias divinidades. De esta manera, el sostén organizativo y moral de una 
sociedad, que es un sostén histórico creado por los mismos humanos que conforman dicha sociedad, aparece como impuesto u ofrecido, por una entidad externa. Las categorías culturales e institucionales son experimentadas casi como biológicas. Y se determinan a partir de principios dicotómicos (Scott 174).

Así, por ejemplo, el Medioevo europeo se constituía como una sociedad ordenada teológicamente a partir de la separación entre lo terrenal y lo divino. La figura del "Hombre", perteneciente a lo terrenal, al universo de la "carne" y marcada por el pecado, estaba destinada al sufrimiento y al sometimiento a las autoridades divinas - en este caso, a la Iglesia y su mundo de clérigos-. Estas se mantenían "puras" a través del mandato de la castidad. El orden social medieval reposaba en la separación estricta entre laicos y clérigos. Los primeros eran percibidos -y se autopercibían-, a través del lente de la Iglesia, como humanos de una naturaleza inferior y corrupta (Wynter, "Unsettling" 259). Los clérigos, a partir de su lugar social y a partir de su comportamiento -con prácticas como el celibato y la abstinencia-, representaban el modelo de lo humano. Estamos hablando entonces de un periodo marcado por la siguiente característica fundamental: el orden económico, político y social estaba basado en la intermediación de lo divino, como fuente y justificación de la estratificación de la sociedad. La Iglesia se presentaba como una institución supranatural, cuyo fundamento no era de este mundo.

Esta división podría entenderse a partir de la categorización de lo no-homogéneo. Es decir, la separación radica en la no-homogeneidad entre el "mundo terrenal" y el "mundo divino" (Wynter, "Unsettling" 275). Se trata de dos órdenes de realidad que no son continuos o, más precisamente, cuyas esencias difieren. Wynter señala que esta discontinuidad estaba también inscrita en el ámbito geográfico. Existía la creencia en una separación entre las "tierras habitadas" y la "zona tórrida" donde la vida humana sería imposible. Esta creencia -representada incluso gráficamente en los mapas de la época- ordenaba la percepción del mundo. Entender esta organización de la sociedad y su principio explicativo permite entonces comprender la concepción que Wynter tiene del humanismo.

Por esto, podemos afirmar que el humanismo, en un sentido amplio, se compone de las distintas figuras de lo humano que han aparecido en las narrativas que sustentan el orden simbólico de Occidente. Se puede 
afirmar que la primera definición a la que Wynter se refiere es la que surge con el Renacimiento. En efecto, para ella, la clave humanista se entiende en la medida en que los intelectuales del siglo XV transformaron la visión de lo humano, rompiendo con el legado medieval. Este orden simbólico que acabamos de definir siguiendo los pasos de Wynter, de manera sucinta, es el orden que es trastocado por la revolución intelectual del Renacimiento, la cual tiene en Pico della Mirandolla a uno de sus principales exponentes. En su Discurso sobre la dignidad del hombre, della Mirandolla escribe:

Oh Adán, no te he dado ni un lugar determinado, ni un aspecto propio, ni una prerrogativa peculiar con el fin de que poseas el lugar, el aspecto y la prerrogativa que conscientemente elijas y que de acuerdo con tu intención obtengas y conserves. La naturaleza definida de los otros seres está constreñida por las precisas leyes por mí prescritas. Tú, en cambio, no constreñido por estrechez alguna te la determinarás según el arbitrio a cuyo poder te he consignado (4).

La reescritura del Génesis ubica al ser humano en un nuevo rol. No ya como ser maldito por el pecado original, sino como actor libre, cuya voluntad está dirigida hacia el control y al conocimiento de la naturaleza. Este es el primer sentido que debe atribuirse al término "humanismo". Wynter incluso lo define como un "humanismo cívico" ("Unsettling" 277). Este está ligado al surgimiento histórico del Estado absolutista. En efecto, el nuevo concepto de "hombre" se traduce en un ser que sigue y obedece las leyes del Estado y no como un ser que busca la redención de la carne a través del espíritu. Wynter afirma:

It was therefore to be on the basis of this new conception, and of its related civic-humanist reformulation, that Man was to be invented in its first form as the rational political subject of the state, as one who displayed his reason by primarily adhering to the laws of the state ${ }^{9}$ (ibid.).

9 "Iba a ser, entonces, sobre la base de esta nueva concepción, y de su correspondiente reformulación cívico-humanista, que el Hombre sería concebido en su forma primera como el sujeto político-racional del Estado, como alguien que exhibía su razón principalmente al adherir a las leyes del Estado" (Traducción del editor). 
El mundo aparece entonces como un mundo creado para el "Hombre", cuyas reglas y leyes físicas pueden ser conocidas por él. El mundo tiene que ser cognoscible. Con el Renacimiento y la Revolución de Copérnico, así como el inicio de la expansión imperial de Europa, se da lo que Wynter llama la emergencia de un mundo sin dios (Wynter, "Unsettling" 311). En otras palabras, son principios seculares los que van a reorganizar la estructura social y van a asentar las categorías a partir de las que se define lo humano. Es el "Hombre", entonces, el que comienza a interpretar en la Naturaleza -como creación divina- las leyes que rigen al mundo y puede entonces desarrollar las ciencias naturales. De esta manera, por ejemplo, la comprensión no-homogénea del mundo se cae con la física de Newton, rompiendo así con la representación de un espacio superior divino y de un espacio inferior terrenal (Scott 195). Wynter plantea que, de esta forma, se inaugura "el milenio del Hombre" (Scott 165; Wynter, "The Ceremony" 22).

Con la llegada de Colón a las costas de lo que se denominará luego "el Caribe", ocurre también una ruptura en la concepción del espacio. Si antes de la exploración hacia el oeste, se pensaba en la existencia de antípodas -es decir, de tierras no habitables-, la aventura colombina desarticula paulatinamente dicha creencia, extendiendo la ecúmene. Ahora bien, este evento histórico, que inaugura el imperialismo europeo, plantea el enfrentamiento de la concepción renacentista del "Hombre" al Otro colonial. El Caribe emerge como parte de dicha ruptura, donde se plantea esta nueva visión del "Hombre". El límite del humanismo cívico aparece entonces claramente cuando se instaura una nueva lógica de poder que somete a los cuerpos, que somete las mentes y que destruye todo aquello que amenaza o inquieta su propia autoconcepción. El modelo del "Hombre" es el modelo del hombre europeo, cristiano y blanco, cuyo pendiente político, es la afirmación del absolutismo estatal.

El siglo XVI es el terreno de esta transformación. La tensión entre la concepción cristiana del humano y la nueva concepción del "Hombre" se plantea en la disputa entre Ginés de Sepúlveda y Bartolomé de Las Casas. La esencia de la Controversia de Valladolid implica la imposición de un nuevo concepto del "Hombre" cuyas raíces van a ser tomadas de la filosofía griega. Es la posibilidad de definir la naturaleza de lo humano a partir de la racionalidad y el sometimiento a la ley natural, así como la reactualización de la oposición entre "bárbaros" y "civilizados", lo que 
termina por imponer la visión secular del modelo del Hombre. En la Controversia, dos antropologías se enfrentan, dos postulados de lo que es el humano. El nuevo criterio de la razón reemplaza a la redención del espíritu, operando un cambio en el "código maestro" de la vida y la muerte simbólicas (Wynter, "Unsettling” 287) ${ }^{10}$. El triunfo empírico de las tesis de Sepúlveda instaura una división entre los "humanos" y los "no-humanos" o "subhumanos". Estos últimos encarnados en los pueblos dominados y racializados (indios y negros).

El siglo XVIII, nos dice Wynter, agrega un criterio más a la comprensión y a la definición del "Hombre". Se trata de la racionalidad, pero esta vez sin referencia alguna al ámbito de la religión. La separación entre "irracionales" y "racionales" será un principio de ordenamiento social en el espacio europeo, como bien lo muestra Foucault, con el internamiento de los locos (Histoire 67). Mientras tanto, en el espacio colonial, dicha dicotomía va a legitimar el sometimiento del Otro colonial. El "nativo", el "negro" y el "indio" son lanzados por lo tanto a los márgenes de lo humano. La siguiente fase del expansionismo imperial terminará por ordenar su entera exclusión, ubicándolos en lo infrahumano (Wynter, "The pope” 26).

En efecto, nos dice Wynter, el imperialismo triunfal y la hegemonía europea utilizará la nueva teoría de Darwin sobre la evolución como un arma social y como base fundamental para instaurar los nuevos principios del mundo moderno. El triunfo de la teoría de la evolución como marco interpretativo de lo natural vino a superar la división entre "irracionalidad" animal y "racionalidad humana". También permitió extraer el origen del mundo, y a los seres humanos, de su causa sobrenatural. Sin embargo, introdujo, paradójicamente, nuevos criterios para modelar al "Hombre" (Scott 204). La oposición entre "evolucionado" y "no-evolucionado", permite establecer una serie de jerarquías dentro de la humanidad, a partir de criterios seudobiológicos, que se cristalizan

10 Esta parte de los trabajos de Wynter tiene el influjo claro de los pensadores de la inflexión decolonial. Sobre todo de aquellos radicados en Estados Unidos -como W. Mignolo-, y que marcaron a inicios del siglo XXI los debates del Grupo Colonialidad/Modernidad -como Quijano-. Para Wynter, es necesario desmontar las diferentes facetas de la colonialidad - del poder, del ser-para poder destruir el orden simbólico en el cual existimos. Dicho trabajo de destrucción está ligado a la posibilidad de desmontar las definiciones de lo "humano" y del "Hombre" que Occidente ha impuesto. 
en la categoría de "raza". La "raza" se convierte entonces en el código a través del cual se "sabe", se "define" lo que es lo humano, pero también a través del cual se "experimenta" la humanidad. Por eso, la experiencia del colonizado pasa principalmente por la definición de su raza, que lo excluye de inmediato del modelo de lo humano (Wynter, "The Pope" 30; Marriot 46). Este punto permite observar cómo Wynter incorpora el trabajo de otros autores caribeńos que han tratado y criticado el tema del humanismo.

En efecto, autores como Césaire, nos dice Wynter, observaron las contradicciones del humanismo cuyo relato colapsó en el siglo XX y la Segunda Guerra Mundial. El Discurso sobre el colonialismo muestra los callejones sin salida del relato humanista europeo. Un humanismo vacío, centrado en el hombre blanco y desprovisto de perspectiva para con los pueblos dominados y colonizados. Un relato que lanza a dichos pueblos al ámbito del "no-ser". Para Wynter, se trata de una primera condena del "humanismo", en su versión finisecular. Césaire plantea a su modo la historicidad de nuestros modos de subjetividad. Muestra cómo se plantea la cuestión de la Otredad abyecta en las Antillas (Wynter, "Beyond the Word" 640). Cuestión que va a ser retomada y desarrollada por Fanon (93), quien plantea cómo esta consideración del modelo de lo "Humano" lleva a un autorechazo del colonizado. Autorechazo que supuestamente permite el acceso al ideal. De esto toman consciencia los autores antillanos y, por lo tanto, luchan contra el mimetismo del ser, en términos individuales, y contra el mimetismo cultural, en términos colectivos (Wynter, "Beyond the Word" 640). En su análisis del Discours antillais, Wynter afirma:

There our "error of reason" with respect to the premise of an absolute "human nature", as one based on our belief in a relation of pure continuity between the genetically regulated modes of organic life and the always rhetorico-discursively regulated modes of human life, still represents our present model of the human. [...] The war waged by the new discourse of the Antilles is a war waged against that error ${ }^{11}$ (Wynter, "Beyond the Word" 645)

11 'Allí nuestro 'error de razón' con respecto a la premisa de una 'naturaleza humana' absoluta, como una basada en nuestra creencia de una relación de total continuidad entre los modos genéticamente regulados de vida orgánica y los modos siempre retórico-discursivamente regulados de la vida humana, todavía representa 
De esta manera, Césaire -revalorizando al nègre-, Fanon -pasando de la ontogénesis a la sociogénesis-y Glissant - proyectando l'étant en lugar de l'Être y reivindicando la especificidad de la historia antillanarefutan el modelo de lo humano absoluto y atemporal.

La crítica de Wynter se inscribe entonces en esta tradición caribeña. Sin embargo, pretende desmontar la constitución misma de la imagen del "Hombre" que gobierna nuestras formas de entender el mundo y nuestra cotidianidad existencial. Por eso, traza la historia de dicha construcción y sus diferentes facetas. Para Wynter, se trata también de demostrar el dominio de esta última fase del "humanismo", construida a través de una imposición de la figura del "Hombre" como el espécimen más acabado y perfecto de la evolución. "Hombre" que encarna el ideal del capitalismo consumista y depredador de recursos y, por lo tanto, la amenaza viva a nuestra sobrevivencia como especie.

De esta manera, el humanismo, tanto en su versión renacentista como en su vertiente evolucionista, es únicamente una imposición del modelo del "Hombre". Dicho modelo se corresponde con lo que Europa -en su despliegue militar, colonial, pero también epistémico y científico- ha definido. Cada una de estas perspectivas humanistas, nos dice Wynter, plantea un orden de conocimiento. Es decir, es dentro de dichas perspectivas y solamente acorde con ellas, que se definen los límites de lo cognoscible, a la vez que de lo aceptable. Ahora bien, ¿qué consecuencias trae esta crítica?, ¿se debe por lo tanto renunciar al "humanismo"?, ¿o es posible un reencanto del mismo?

\section{¿QUÉ HACER CON EL HUMANISMO?}

La crítica de Wynter tiene una característica que podemos encontrar en las elaboraciones de pensadores caribeños, tal y como lo señalamos anteriormente. Sin embargo, está nutrida también por una fina lectura de la obra de Foucault de la cual Wynter retoma la noción de episteme, que explica la extensión y la dimensión de lo cognoscible para cada época (Les mots 314). Wynter también retoma la tesis foucaultiana de una "invención del Hombre" (355). Sin embargo, extiende el entendi-

nuestro modelo actual de lo humano [...] La guerra librada por el nuevo discurso de las Antillas es una guerra librada contra aquel error" (Traducción del editor). 
miento de esta construcción más allá del periodo histórico que Foucault analiza, y aprovecha el encuadre desarrollado por el filósofo francés para mostrar cómo se organizan una serie de saberes para legitimar los fundamentos de la sociedad y del orden. Ahora bien, para un autor como Foucault, el significante "Hombre" representa ante todo un problema epistemológico y de construcción del conocimiento. En cambio, los pensadores coloniales ven ahí la justificación de los más horrendos crímenes. Detrás del "Hombre" se desata, en el ámbito colonial, una destrucción del ser humano concreto. Por lo tanto, más que un proceso inacabado, el humanismo tanto en su versiòn renacentista como en su versión evolucionista, sería un fracaso consumado.

Pero Wynter busca responder a esta interrogante a partir de la puesta en evidencia del funcionamiento de cada uno de estos cambios antes descritos. En efecto, el paso de lo que podríamos llamar un sistema de sentido hacia otro sistema de sentido, se realiza por un cambio sustancial que permite una ruptura. Dicha ruptura tiene algo de revolucionaria y es en cierta medida la idea que surge fuera de los límites del campo de comprensión existente. Ahora bien, el trabajo principal de Wynter consiste entonces en hacer visibles las bases invisibles sobre las cuales se sostiene nuestro sistema actual, nuestro orden de conocimiento o -para entenderlo en otros términos- nuestra forma de "estar en el mundo". De ahí la importancia del enfoque histórico que va analizando cómo se construyen los parámetros de sentido, a partir de los cuales definimos al "Hombre", pero también, a partir de los cuales entendemos la Historia (Marriot 47).

Así, la situación contemporánea, anclada en esta forma -no ya de un discurso sobre el humanismo, sino en la imposición de un modelo del "Hombre"- puede ser descrita como el sometimiento de todas las formas de vida y de organización social, a ese único modelo. Entiéndase aquí, un hombre blanco, heterosexual, burgués, propietario perteneciente a una minoría numérica. El desafío es, pues, la ruptura con este orden de conocimiento, con esta estructura social que produce, sin cesar, vidas humanas fuera del modelo y, por lo tanto, desechables (Wynter, "Tras el 'Hombre" 75; Scott 190). Para Wynter, salvar el "humanismo" es demostrar que su construcción histórica ha sido la tentativa de universalizar, por la fuerza, una experiencia humana particular. Y, por lo tanto, pensar otro "humanismo" es darles espacio a esas otras 
experiencias de lo humano. Es enfrentar, nos dice Wynter, al "Hombre" con lo humano ("1492: Una nueva visión” 415), con el fin de salvar la especie y abandonar al "Hombre".

De esta manera, resulta necesario evidenciar que la concepción del humanismo en el que hemos sido formados, es una concepción etnoclasista, tanto en su vertiente renacentista como en su variante evolucionista. Y así como fue imperativo, en cada salto o cambio epistemológico, reinventar la identidad social propia, fuera de los límites de la concepción previa, es ahora fundamental reinventar una identidad social, basada en un "humanismo ecuménico". Es decir, en un humanismo que rompa con el modelo de lo humano heredado de siglos de dominación occidental. Solo un humanismo con estas características puede verdaderamente ser calificado de universal. Ahora bien, dicho imperativo, según Wynter, no puede ser una ruptura con lo que la modernidad ha traído de positivo. Se debe entender, nos dice Wynter, que el quiebre epistemológico ha logrado, por primera vez en la historia, un mundo sin dios, es decir, la posibilidad misma de la emancipación humana y de la libertad.

Ahora bien, se debe incorporar este legado y sobrepasarlo, a partir de una consideración de lo que nos hace humanos, una praxis de humanidad o más precisamente, una autopoiesis (Wynter, "Towards" 60; Hantel 74). Redefinir a los humanos no como seres bioevolucionados, como lo hemos hecho desde Darwin, sino como un híbrido de "bios" y "logos". Cuando esto se logre, un nuevo modo de ser humano puede pensarse, un modo que no requiera de la oposición entre un "Otro" y un "nosotros". Como un híbrido biocultural que somos, nos imponemos una autopoética. Entendiendo aquí "poética" como una forma de construirnos, una acción a partir de la cual los humanos trabajan para crearse a sí mismos. En este sentido, la propuesta de Wynter retoma la idea que subyace al Discours de Glissant. Se trata de un nuevo discurso que va más allá de la palabra del "Hombre". Se trata de proponer y construir un discurso que está destinado a reemplazar ese modelo de un "Hombre" -sujeto ontogenético- por un sujeto relativo a la cultura y a su forma de estar en el mundo. Si el modelo del "Hombre" se impuso como forma retórico-discursiva y neurofisiológica instituida, es a partir de la liberación de la palabra propia -una poética como la que postula Glissant- que puede destronarse ese orden y construirse un orden alternativo. Y este trabajo requiere la fundamentación de una nueva ciencia 
de lo humano que pueda romper los límites de la distinción disciplinar. Esfuerzo preliminar, nos dice Wynter, para toda tentativa de cambio social ("Beyond the Word" 647).

\section{Conclusiones}

De esta manera, para Wynter, hemos de dirigirnos hacia una nueva definición de lo humano, como especie, que rompa con el esquema heredado de esta fase de neoliberalismo capitalista, que rompa con la imagen del Hombre como aquella de la burguesía corporativa financiera occidental. Wynter considera que el rol de la academia en este sentido es fundamental, puesto que es ahí donde se debe dejar al descubierto estas estructuras inconscientes que sostienen todo el aparato del saber, que regulan nuestros deseos y nuestra forma de acercarnos al mundo. Es ahí, a partir de dichos espacios y con la difusión de un saber crítico que se debe desmontar la episteme que literalmente nos gobierna (Wynter, "No Humans" 46). Una episteme que reproducimos como garantes de un saber institucionalizado que es incapaz de interrogar sus propios a priori. Se trata de completar una revolución humanista que está inacabada. En este sentido, el anhelo de Wynter va de la mano con la propuesta de Césaire de fundar una "nueva ciencia del Mundo", que localice nuestra humanidad no solo en las realidades de la carne y la actividad neuronal, sino también en nuestra capacidad fundamental para narrar nuestros diversos mundos sociales y hacerlos existir (Bayani 835). O en otras palabras, será "a reinvented studia humanitatis or a science of the human, which takes as its object of inquiry the correlation between our rhetoric discursively instituted systems of meanings and the neurochemical signaling field that they orchestrate"12 (Wynter, "Beyond the Word" 646).

Con esto en mente, este nuevo concepto de lo humano está llamado a realizarse plenamente en la medida de toda su autonomía.

12 "Un studia humanitatis reinventado o una ciencia de lo humano que tome como su objeto de investigación la correlación entre nuestros sistemas de significado establecidos retórico-discursivamente y el campo de señalización neuroquímica que estos orquestan" (Traducción del editor). 
BibLIOGRAFÍA

Bayani Rodriguez, Anthony. "Introduction: On Sylvia Wynter and the Urgency of a New Humanist Revolution in the Twenty-First Century". American Quarterly, vol. 70, N 4, 2018, pp. 831-836.

Brathwaite, Kamau. "Caliban, Ariel and Unprospero in the conflict of creolization". Annals of the New York Academy of Sciences, vol. 292, $\mathrm{N}^{\circ} 1,1977$, pp. 41-61.

Benítez-Rojo, Antonio. La isla que se repite. Barcelona, Editorial Casiopea, 1998.

CÉsaire, Aimé. “Discurso sobre el colonialismo”. Guaraguao, № 20, 2005, pp. 157-193.

Della Mirandola, Giovanni Pico. Discurso sobre la dignidad del hombre. Ciudad de México, UNAM, 2004.

Dubois, William Edward Burghardt. The Souls of Black Folk. Greenwich, Fawcett, 1961.

Edwards, Norval. "Talking about a little culture: Sylvia Wynter's Early Essays". Journal of West Indian Literature, vol. 10, $\mathrm{N}^{\circ} 1-2$, 2001, pp. 12-38.

Fanon, Frantz. Piel negra, máscaras blancas. Barcelona, Akal, 2009.

Foucault, Michel. Histoire de la folie à l'âge classique. París, Gallimard, 1976. . Les mots et les choses. París, Gallimard, 1966.

Glissant, Édouard. Le discours antillais. París, Folio, 2002.

Goveia, Elsa. "Conclusión" (Estudio de la historiografía de las Antillas inglesas hasta finales del siglo XIX). Antología de Pensamiento Crítico Caribeño, Félix Valdés García (comp.), Buenos Aires: CLACSO, 2017, pp. 59-70.

Hantel, Max. "What Is It Like to Be a Human?: Sylvia Wynter on Autopoiesis". PHILOSOPHIA, vol. 8, N 1, 2018, pp. 61-79.

Marriott, David. "Inventions of Existence: Sylvia Wynter, Frantz Fanon, Sociogeny, and 'the Damned'". The New Centennial Review vol. 11, N³, 2012, pp. 45-89. 
McKittrick, Katherine. Sylvia Wynter: On being human as a Praxis. Durhan y Londres, Duke University Press, 2015.

Scott, David. "The Re-Enchantment of Humanism: An Interview with Sylvia Wynter". Small Axe, No 8, 2000, pp. 119-207.

Torres Saillant, Silvio. An Intellectual History of the Caribbean. Londres, Palgrave Macmillan, 2006.

Thomas, Greg. “The Body Politics of 'Man' and 'Woman' in an 'Anti-Black' World: Sylvia Wynter on Humanism's Empire (A Critical Resource Guide)". On Marronage: Ethical Confrontations with Anti-Blackness, Khalil Saucier y Tryon P. Woods (eds.), Nueva Jersey, Africa World Press, 2015, pp. 67-107.

Wynter, Sylvia. "Lady Nugent's Journal". Jamaica Journal, vol. 1, $\mathrm{N}^{\circ} 1,1967$, pp. 23-34.

. "Jonkonnu in Jamaica: Towards the Interpretation of Folk Dance as a Cultural Process". Jamaica Journal No 4, 1970, pp. 34-38.

. "Novel and History, Plot and Plantation". Savacou, N ${ }^{\circ} 5$, 1971, pp. 95-102.

. "One Love - Rhetoric or Reality? - Aspects of Afro-Jamaicanism". Caribbean Studies, N 12, 1972, pp. 64-99.

. "The Ceremony Must Be Found: After Humanism". Boundary, vol. 2, N³/13, 1984, pp. 19-70.

. "Beyond the Word of Man: Glissant and the New Discourse of the Antilles". World Literature Today, vol. 63, № 4, 1989, pp. 637-48.

. "No Humans Involved”. Forum N.H.I., N 1, 1994, pp. $42-74$.

. "The Pope must have been drunk, the King of Castille a Madman: Culture as Actuality, and the Caribbean rethinking Modernity". Reordering of Culture: Latin America, the Caribbean and Canada in the Hood, Alvina Ruprecht y Cecilia Taiana (eds.), Ottawa, Carleton University Press, 1995, pp. 17-41.

. "Towards the Sociogenic Principle: Fanon, Identity, the Puzzle of Conscious Experience". National Identities and Socio-Political Changes in Latin America, Antonio Gomez-Moriana y Mercedes F. Duran-Cogan (eds.), Nueva York, Routledge, 2001, pp. 30-66, 
. "Unsettling the Coloniality of Being/Power/Truth/Freedom: Towards the Human, After Man, Its Overrepresentation-An Argument". The New Centennial Review, No 3, 2003, pp. 257-337. . "Tras el 'Hombre', su última palabra: sobre el posmodernismo, les damnés y el principio sociogénico". La teoría política en la encrucijada descolonial, Walter Mignolo (ed.), Buenos Aires, Ediciones del Signo, 2009, pp. 51-123.

. "1492: Una nueva visión del mundo". Antología de Pensamiento Crítico Caribeño, Félix Valdés García (comp.), Buenos Aires, CLACSO, 2017, pp. 367-425.

White, Derrick. "Black Metamorphosis: A Prelude to Sylvia Wynter's Theory of the Human". The C. L. R. James Journal, vol. 16, $\mathrm{N}^{\circ} 1$, 2010, pp. 127-48.

Recepción: 30-09-20

Aceptación: 31-12-20 